INSTITUT NATIONAL DE RECHERCHE EN INFORMATIQUE ET EN AUTOMATIQUE

\title{
A non-linear observer for unsteady three-dimensional flows
}

\author{
Marcelo Buffoni — Simone Camarri — Angelo Iollo — Edoardo Lombardi — Maria \\ Vittoria Salvetti
}

\section{$\mathbf{N}^{\circ} ? ? ? ?$}

Février 2007

Thème NUM

\section{apport \\ de recherche}





\title{
RINRIA
}

\section{A non-linear observer for unsteady three-dimensional flows}

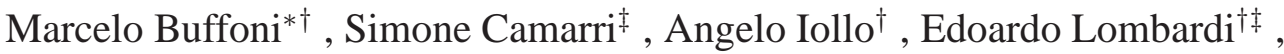 \\ Maria Vittoria Salvetti ${ }^{\ddagger}$
}

Thème NUM - Systèmes numériques

Projet MC2

Rapport de recherche $\mathrm{n}^{\circ}$ ???? — Février 2007 -22 pages

\begin{abstract}
A method is proposed to estimate the velocity field of an unsteady flow using a limited number of flow measurements. The method is based on a non-linear low-dimensional model of the flow and on expanding the velocity field in terms of empirical basis functions. The main idea is to impose that the coefficients of the modal expansion of the velocity field give the best approximation to the available measurements and that at the same time they satisfy as close as possible the nonlinear low-order model. The practical use may range from feedback flow control to monitoring of the flow in non-accessible regions. The proposed technique is applied to the flow around a confined square cylinder, both in two- and three-dimensional laminar flow regimes. Comparisons are provided with existing linear and non-linear estimation techniques.
\end{abstract}

Key-words: low-order models, dynamic estimation, non-linear observers

\footnotetext{
* DIASP - Politecnico di Torino, 10129 Torino, Italy

${ }^{\dagger}$ MAB - Université Bordeaux 1 et MC2 - INRIA Futurs, 33405 Talence, France

‡DIA - Università di Pisa, 56127 Pisa,Italy
} 


\section{Un observateur non linéaire pour des écoulements tridimensionnels instables}

Résumé : Nous proposons une méthode pour estimer le champ de vitesse d'un écoulement instable en utilisant un nombre limité de mesures. La méthode est basée sur un modèle d'ordre réduit non linéaire de l'écoulement et sur l'expansion du champ de vitesse en termes de fonctions de base empiriques. L'idée principale est d'imposer que les coefficients de l'expansion modale du champ de vitesse donnent la meilleure approximation des mesures disponibles et qu'en même temps ils satisfassent aussi étroitement que possible le modèle d'ordre réduit non linéaire. L'utilisation pratique peut s'étendre du contrôle actif en boucle fermée de l'écoulement à la surveillance de l'écoulement dans des régions inaccessibles. La technique proposée est appliquée à l'écoulement autour d'un cylindre carré confiné, dans des régimes laminaires bi et tridimensionnels. Des comparaisons avec des techniques linéaires et non linéaires d'évaluation existantes sont fournies.

Mots-clés : modèles réduits, estimation dynamique, observateurs non linéaires 


\section{Introduction}

The problem of deriving an accurate estimation of the velocity field in an unsteady complex flow, starting from a limited number of measurements, is of great importance in many engineering applications. For instance, in the design of a feedback control, a knowledge of the velocity field is a fundamental element in deciding the appropriate actuator reaction to different flow conditions. In other applications it may be necessary or advisable to monitor the flow conditions in regions of space which are difficult to access or where probes cannot be fitted without causing interference problems.

The method that we propose exploits an idea which is similar to that at the basis of the Kalman filter (see [11]). The starting point is a Galerkin representation of the velocity field $\boldsymbol{u}(\boldsymbol{x}, t)$ in terms of $N_{r}$ empirical eigenfunctions, $\boldsymbol{\Phi}^{i}(\boldsymbol{x})$, obtained by Proper Orthogonal Decomposition (POD) (see [12])

$$
\boldsymbol{u}(\boldsymbol{x}, t)=\overline{\boldsymbol{u}}(\boldsymbol{x})+\sum_{i=1}^{N_{r}} a_{i}(t) \boldsymbol{\Phi}^{i}(\boldsymbol{x})
$$

where $\boldsymbol{u}(\boldsymbol{x}, t): \mathbb{R}^{n} \times[0, T] \rightarrow \mathbb{R}^{n}, \Phi^{i}(\boldsymbol{x}): \mathbb{R}^{n} \rightarrow \mathbb{R}^{n}, n \in\{2,3\}$ according to the physical space dimension, $\overline{\boldsymbol{u}}(\boldsymbol{x})$ is some reference velocity field and $a_{i}(t): I=[0, T] \subset \mathbb{R} \rightarrow \mathbb{R}$.

For a given flow, the POD modes can be computed once for all based on Direct Numerical Simulation (DNS) or on highly resolved experimental velocity fields, such as those obtained by particle image velocimetry. An instantaneous velocity field can thus be reconstructed by estimating the coefficients $a_{i}(t)$ of its Galerkin representation.

One simple approach to estimating the POD coefficients is to approximate the flow measurements in a least square sense, as done, for instance, in [9].

A similar procedure is also used in the estimation based on gappy POD, see [18] and [19]. Another possible approach, the linear stochastic estimation (LSE), is based on the assumption that a linear correlation exists between the flow measurements and the value of the POD modal coefficients (see, for instance, [2]).

However, these approaches encounter difficulties in giving accurate estimations when threedimensional flows with complicated unsteady patterns are considered, or when a very limited number of sensors is available. Under these conditions, for instance, the least squares approach cited above (LSQ) rapidly becomes ill conditioned. This simply reflects the fact that more and more different flow configurations correspond to the same set of measurements. To circumvent those problems, many contributions in the literature have aimed to determine the effective placement of the sensors (see e.g. [14], [6], [5], [19]). For example in [19], a systematic approach to sensor placement is formulated within the gappy POD framework using a condition number criterion.

In order to improve estimation performance, extensions of the above methods have been proposed: quadratic stochastic estimation (QSE) [1], [13] and spectral linear stochastic estimation (SLSE) [7]. They allow more accurate estimations compared with LSQ or LSE methods, but, in fact, neither of these methods takes into account the underlying dynamic model that the POD coefficients must satisfy, i.e., a finite dimensional equivalent of the Navier-Stokes equations that is obtained by the Galerkin projection of the flow equations on the POD modes retained for the representation of the velocity field. In this sense, the aim of the present study is to discuss an approach that combines a linear estimation of the coefficients $a_{i}(t)$ with an appropriate non-linear low-dimensional flow 


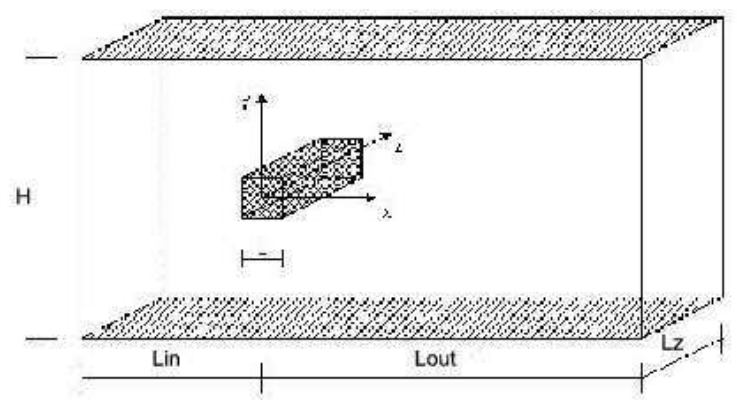

Figure 1: Computational domain $\Omega$.

model. Our objective is not, however, to propose an estimation method that can be readily implemented for real time applications, even if a few indications in this direction are given. Rather, our objective is to understand whether a non-linear observer outperforms existing linear flow observers, without the constraints imposed by an actual recursive algorithm, e.g., a real-time computation. Moreover, instead of what was done, for example, in [10], this study is confined to a deterministic framework, since the model as well as the measurements are supposedly not affected by noise. If, within this framework, a dynamic estimation turns out to be less satisfactory than static approaches, i.e., those which use no model, then there would be little interest in pursuing the research in this direction. In addition, we address the issue of the sensitivity of the proposed approach to sensor type and location. Finally, we present an application to a flow, which is characterized by a significant three-dimensionality and a non-periodic dynamics.

\section{Flow set up and low order model}

The flow over an infinitely long square cylinder symmetrically confined by two parallel planes is considered. A sketch showing the geometry, the frame of reference and the adopted notation is plotted in figure 1. At the inlet, the incoming flow is assumed to have a Poiseuille profile with maximum center-line velocity $U_{c}$. Two Reynolds numbers $R e=U_{c} L / \nu$ were considered, one at which the flow is two-dimensional $(R e=150)$ and the other one leading to a three-dimensional flow in the wake $(R e=300)$. With reference to figure $1, L / H=1 / 8, \operatorname{Lin} / L=12, \mathrm{Lout} / L=$ 20 and $L z / L=0.6$ for the two-dimensional case, whereas $L / L z=6$ for the three-dimensional one. Periodic boundary conditions are imposed in the span-wise direction and no-slip conditions are enforced both on the cylinder and on the parallel walls. Details concerning the grids and the numerical set up are reported in [3]. All the quantities reported in the following have been made non-dimensional by $L$ and $U_{c}$. The two-dimensional flow obtained at $R e=150$ is a classic vortex street with a well defined shedding frequency. However, the interaction with the confining walls adds to the complexity of the flow and leads to some peculiar features, like the fact that the vertical position of the span-wise vortices is opposite to the one in the classic von Kármán street ([4]). For 
the three-dimensional case the situation is even more complex, due to instabilities developing in the span-wise direction. The flow is no longer periodic and exhibits complicated flow patterns ([3]).

The POD modes $\boldsymbol{\Phi}^{k}(\boldsymbol{x})$ are found using the snapshot method ([15])

$$
\boldsymbol{\Phi}^{k}=\sum_{i=1}^{N} b_{i}^{k} \boldsymbol{U}^{(i)}
$$

where $\boldsymbol{U}^{(i)}=\boldsymbol{u}\left(\boldsymbol{x}, t_{i}\right)$ are flow snapshots taken at time $t_{i} \in[0, T], N$ is the number of snapshots, $k \in\{1, \ldots, N\}$, and the coefficients $b_{i}^{k} \in \mathbb{R}$ are such that the vectors $\left(b_{1}^{k}, \ldots, b_{N}^{k}\right)$ are the eigenvectors of the time correlation matrix $\int_{\Omega} \boldsymbol{U}^{(j)} \cdot \boldsymbol{U}^{(l)} d x$, of size $N \times N$. Only a limited number of modes, $N_{r}$, is used to represent the velocity field. In particular we took $N_{r}=6$ and $N_{r}=20$ for the two-dimensional and the three-dimensional cases, respectively.

A Galerkin projection of the incompressible Navier-Stokes equations over the retained POD modes has been carried out. This leads to the following $N_{r}$-dimensional dynamical system

$$
\begin{aligned}
& R_{r}(\boldsymbol{a}(t))=\dot{a}_{r}(t)-A_{r}-C_{k r} a_{k}(t)+B_{k s r} a_{k}(t) a_{s}(t)=0 \\
& a_{r}(0)=\left(\boldsymbol{u}(\boldsymbol{x}, 0)-\overline{\boldsymbol{u}}(\boldsymbol{x}), \boldsymbol{\Phi}^{r}\right)
\end{aligned}
$$

where $\boldsymbol{a}(t): I \rightarrow \mathbb{R}^{N_{r}}$ and $\boldsymbol{a}(t)=\left\{a_{1}(t), \ldots, a_{N_{r}}(t)\right\} ; r, k$ and $s$ run from 1 to $N_{r}$ and the Einstein summation convention is used. The scalar coefficients $B_{k s r}$ come directly from the Galerkin projection of the non-linear terms in the Navier-Stokes equations, and they can easily be expressed in terms of the POD modes. The scalar terms $A_{r}$ and $C_{k r}$ are calibrated using a pseudo-spectral method to take into account the pressure drop, as well as the interaction of unresolved modes in the POD expansion. The calibration consists in solving an inverse problem, where the coefficients $A_{r}$ and $C_{k r}$ are found in order to minimize the difference, measured in the $L^{2}$ norm, between the model prediction and the actual reference solution. See [8] for a detailed discussion of the calibration technique.

The resulting model for the two-dimensional flow configuration considered here is very accurate in describing the asymptotic attractor ([9] and [8]). For the three-dimensional case, it was shown in [3] that the calibrated model is capable of accurately reproducing the complicated flow dynamics resulting from the interaction of the three-dimensional vortex wake with the confining walls inside the calibration interval. This is shown in figure 2, where the predictions of some POD modal coefficients given by the dynamic POD model within the calibration interval are compared to those obtained from the projection of the fully resolved Navier-Stokes simulations. However, these results rapidly deteriorate as soon as the model becomes an actual prediction tool, i.e., outside the time interval in which the calibration is performed. Nonetheless, the model can be used in conjunction with experimental measurements in order to reconstruct the entire flow field, as will be shown in the next section.

\section{Non-linear observer}

Our aim is to provide an estimation of the modal coefficients $a_{i}(t)$ starting from $N_{s}$ flow measurements $f_{k}, k \in\left\{1, \ldots, N_{s}\right\}$. Let $\bar{\alpha}_{i}(t)$ be the projection of the velocity field $\boldsymbol{u}(t)$ over the $i$-th POD mode and $\alpha_{i}(t)$ be its estimated value at time $t$.

RR $n^{\circ} 0123456789$ 

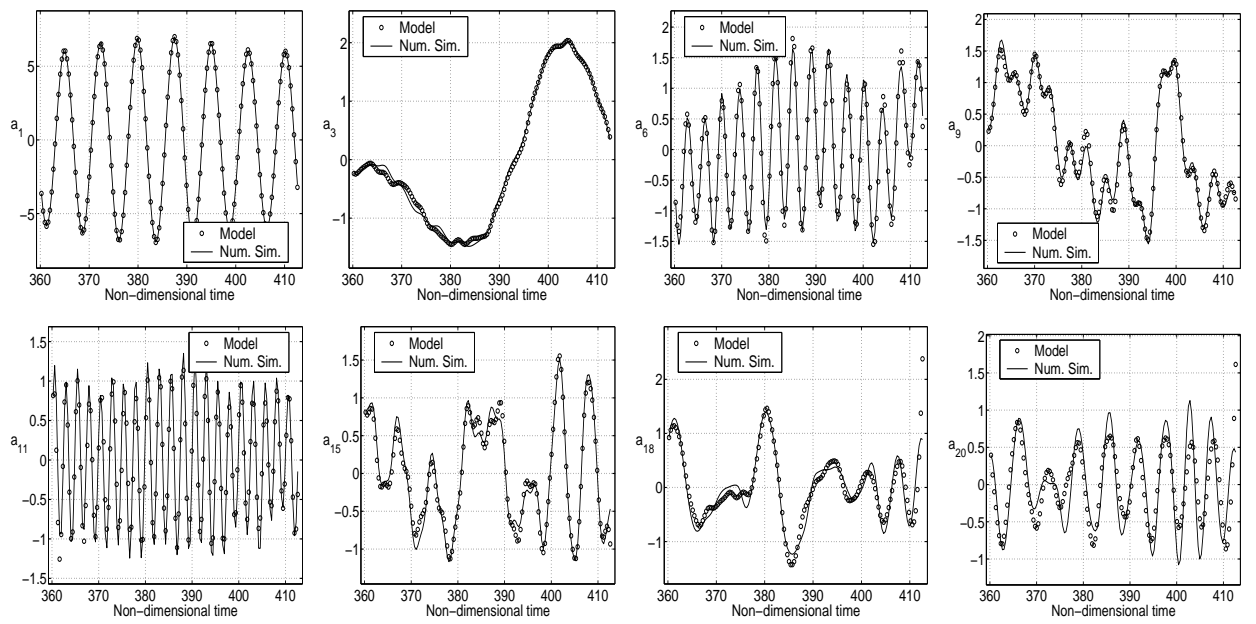

Figure 2: Three-dimensional flow: projection of the fully resolved Navier-Stokes simulations over the POD modes (continuous line) vs. the integration of the dynamical system inside the calibration interval, obtained retaining the first 20 POD modes (circles). Only 8 representative modal coefficients are shown here.

We assume that each measurement $f_{k}$ is a scalar quantity which depends linearly on the instantaneous velocity field $\boldsymbol{u}(t)$. For instance, $f_{k}$ can be a point-wise measurement of a velocity component, or of a shear stress, or it can be a spatial average of a linear combination of velocity components.

The available spatial information may be exploited by using a LSQ approach, as done in [9]. At any given time $\tau$, thanks to the linearity of $f_{k}$ with respect to $\boldsymbol{u}$ and to the modal decomposition of the velocity field (see Eq. (1)), $f_{k}$ can be written in terms of POD modes

$$
f_{k}(\boldsymbol{u}(\tau)) \simeq \sum_{j=1}^{N_{r}} a_{j}(\tau) f_{k}\left(\Phi^{j}\right)
$$

where $f_{k}\left(\boldsymbol{\Phi}^{j}\right)$ is obtained from the application of $f_{k}$ to the vector field associated to mode $\boldsymbol{\Phi}^{j}$. Then, the following least-squares problem has to be solved for every $\tau$

$$
\min _{\left\{a_{1}(\tau), \ldots, a_{N_{r}}(\tau)\right\}} \sum_{k=1}^{N_{s}}\left(f_{k}(\boldsymbol{u}(\tau))-\sum_{j=1}^{N_{r}} a_{j}(\tau) f_{k}\left(\boldsymbol{\Phi}^{j}\right)\right)^{2}
$$

This problem leads to the solution of a $N_{r}$-dimensional linear system of equations. Once this problem is solved, the estimated modal coefficients can be written

$$
\alpha_{j}(\tau)=\sum_{k=1}^{N_{s}} \Upsilon_{k j} f_{k}(\boldsymbol{u}(\tau))
$$


where $\Upsilon$ is a known rectangular matrix of size $N_{s} \times N_{r}$. The error minimization (44) leads to a linear representation of the estimated modes as a function of the measurements.

The LSE approach, conversely, exploits temporal rather than spatial information and is based on the assumption that a linear relation exists between the modal coefficients and the measurements

$$
\alpha_{j}(\tau)=\sum_{k=1}^{N_{s}} \Lambda_{k j} f_{k}(\boldsymbol{u}(\tau))
$$

where $\Lambda$ is now an unknown rectangular matrix of size $N_{s} \times N_{r}$. This matrix is determined by imposing the condition that $\forall j \in\left\{1, \ldots, N_{r}\right\}$ and $\forall k \in\left\{1, \ldots, N_{s}\right\}$

$$
\int_{0}^{T} \bar{\alpha}_{j}(t) f_{k}(\boldsymbol{u}(t)) d t=\int_{0}^{T} \sum_{m=1}^{N_{s}} \Lambda_{m j} f_{m}(\boldsymbol{u}(t)) f_{k}(\boldsymbol{u}(t)) d t
$$

The time interval $[0, T]$ is the same as that considered for building the POD modes. Hence, since the left-hand side is known, a set of linear equations is obtained; these uniquely define the matrix $\Lambda$.

The LSQ and and LSE both provide linear estimation of the modal coefficients. Matrices $\Upsilon$ and $\Lambda$ have the same size, although the coefficients are different. In the following we overcome the assumption of a linear relation.

Let us assume that a certain number of measurements at consecutive times $\tau_{m}, m \in\left\{1, \ldots, N_{m}\right\}$ are available. The main idea of the dynamic-estimation approach proposed here is to impose that the coefficients of the modal expansion of the velocity field give the best approximation to the available measurements, using either LSQ (5) or LSE (6), and that at the same time they satisfy as closely as possible the non-linear low-order model (2).

In the LSQ case this is done by minimizing the sum of the residuals of (5) and the residuals of (2) for all times $\tau_{m}$. More precisely, let $\boldsymbol{\alpha}(t): \mathbb{R} \rightarrow \mathbb{R}^{N_{r}}$ and $\boldsymbol{\alpha}(t)=\left\{\alpha_{1}(t), \ldots, \alpha_{N_{r}}(t)\right\}$, we have

$$
\boldsymbol{\alpha}(t)=\underset{\boldsymbol{a}(t)}{\operatorname{argmin}} \sum_{m=1}^{N_{m}}\left(C_{R} \sum_{r=1}^{N_{r}} R_{r}^{2}\left(\boldsymbol{a}\left(\tau_{m}\right)\right)+\sum_{r=1}^{N_{r}}\left(a_{r}\left(\tau_{m}\right)-\sum_{k=1}^{N_{s}} \Upsilon_{k r} f_{k}\left(\boldsymbol{u}\left(\tau_{m}\right)\right)\right)^{2}\right)
$$

where $\boldsymbol{a}(t)=\left\{a_{1}(t), \ldots, a_{N_{r}}(t)\right\}$. The parameter $C_{R}$ weights more the measurements (LSQ) or the dynamic model in the definition of the residual norm. It could be systematically tuned, or it could be a matrix. In the numerical experiments reported here, this parameter has been in a heuristic way, leaving a consistent analysis to future investigations. The minimization of this functional is reduced to a non-linear algebraic problem. As in [8], a pseudo-spectral approach is used and each $a_{r}(t)$ is expanded in time using Lagrange polynomials defined on Chebyshev-Gauss-Lobatto collocation points. The necessary conditions for the minimum result in a non-linear set of algebraic equations for the coefficients of the Lagrange polynomials. The solution is obtained by a Newton method, which, in the present applications, usually converges in a few (typically 5 to 8 ) iterations. The solution of the problem (8) provides an estimation for the POD modal coefficients for all modes and for all instants at which measurements are available. This allows the reconstruction of the entire flow field at the same instants through equation (11). The above method, therefore, represents a non-linear observer of the flow state. In the following, it will be referenced as K-LSQ.

RR $n^{\circ} 0123456789$ 
A similar approach can be obtained for the LSE technique, by substituting in Eq. (8) the residuals of Eq. (6) instead of those of Eq. (5). This approach is referenced as K-LSE.

In literature, there exist other flow estimation techniques that are non-linear in the flow measurements. In the following we will compare the results of the proposed non-linear dynamic estimation to one of them, a quadratic extension to LSE ([1], [13]). This method is based on the assumption that equation (6) is just the first term of a Taylor expansion with respect to the sensor measurements, whereas QSE takes into account the second order term, too. Hence, we have

$$
\alpha_{j}(\tau)=\sum_{k=1}^{N_{s}} \Lambda_{k j} f_{k}(\boldsymbol{u}(\tau))+\sum_{k=1}^{N_{s}} \sum_{m=1}^{N_{s}} \Omega_{k m j} f_{k}(\boldsymbol{u}(\tau)) f_{m}(\boldsymbol{u}(\tau))
$$

where the scalar coefficients $\Lambda_{k j}$ and $\Omega_{k m j}$ are obtained using double, triple and quadruple correlations between measurements in an equation equivalent to (7). This approach is referred to as QSE.

Once the matrices appearing in equations (5) (6) and (9) are computed, the estimation of the modal coefficient at a certain time is based on the measurements made at the same time. In contrast, [7], [17] proposed to take into account integrated temporal data by assuming a linear dependence between the modal coefficients and the flow measurements in a non-local way, by working in the frequency domain. Let $\hat{\alpha}$ be the Fourier transform of $\alpha$ and $\hat{f}_{j}$ that of $f_{j}$, then for each frequency we pose

$$
\hat{\alpha}_{j}=\sum_{k=1}^{N_{s}} \hat{\Gamma}_{k j} \hat{f}_{k}
$$

where $\hat{\Gamma}_{k j}$ is a matrix obtained by appropriate ensemble averages and depends on the frequency. In the time domain this amounts to a convolution integral between the measurements and the time dependent matrix $\Gamma$. We call this approach SLSE. As compared to QSE and SLSE, the dynamic estimation procedure that we propose is non-linear and, at the same time, it takes into account the evolution of the modal coefficients in time by constraining such evolution to a model, in the weak sense determined by 8 .

Concerning the applicability of the methods described above, it is important to recall that the LSE and LSQ approaches are readily applicable to real-time estimation, as well as the QSE, although the cost of this last approach scales as $N_{s}^{2}$ instead of linearly as in the previous two cases. Conversely, the SLSE approach is more difficult to be used for real-time estimations, since it uses the whole temporal history of the measurements, collected in a time interval, coupled together (linearly) via the Discrete Fourier Transform (DFT). This implies that the estimation problem must be tackled after having collected enough temporal information and it consists of a number of LSE problems equal to the number of retained frequencies, plus additional DFT's of the measurements and of the estimated POD coefficients.

Similarly to what done in the SLSE approach, in the present dynamic estimations the temporal histories of the measurements are coupled together (non-linearly) by the dynamic POD model. This aspect poses difficulties in a real-time application. Indeed, as pointed out in the introduction, KLSQ or K-LSE are thought to be applied a-posteriori, because their computational cost, although 
unimportant in a post-processing phase, is large for a real-time analysis. Nevertheless, although actual real-time applications are premature, a proposal for their prospective implementation for realtime estimation is the following. The flow state at a given time $t^{*}$ could be estimated by considering the measurements taken at that time and at the previous $N_{m}-1$ ones. At the successive sampling time, the corresponding new measurements are added and the oldest ones are dropped, keeping the number of measurements considered constant. In other words, reconstruction is carried out using a fixed number of measurements distributed in a time interval which is located before $t^{*}$, and which translates as time increases. The sampling rate (i.e. $\tau_{m}-\tau_{m-1}$ ) and $N_{m}$ can be tuned in order to decrease the computational costs while granting the level of accuracy required by the particular application. Moreover, when a new set of measurements is added, the Newton method for solving the non-linear system would be restarted from the previous solution, which is already close to the final solution, thus definitely reducing the number of iterations for convergence.

In contrast with the other methods, the proposed approaches need a working Galerkin model as a fundamental ingredient. The construction of such a model can be carried out from the information needed to build the POD database, a necessary step for all the methods considered here. Therefore no additional information is needed as compared to other approaches.

\section{Results and discussion}

The K-LSQ and K-LSE are used to reconstruct the flow in the configuration described in Sec. 2 , both in the two- $(R e=150)$ and three-dimensional $(R e=300)$ cases. Results are compared to those obtained by the most common techniques available in the literature and cited in the Introduction.

Accuracy in the prediction of the single modal coefficients and in the reconstruction of the velocity fields were appraised. In both cases, differences with respect to the reference case (DNS) were quantified in terms of relative error in the $L^{2}$ norm, i.e., the $L^{2}$ norm of the difference between the estimated and the reference quantity divided by the norm of the reference quantity.

Several parameters are involved in the set-up of the K-LSQ and K-LSE models. They are related to (i) the dynamic POD model: number of retained modes, calibration interval, number and temporal distribution of available snapshots; (ii) the selected flow measurements: number, type and collocation.

The parameters involved in the derivation of the POD dynamical system used herein are the same that were selected in previous studies $([\overline{9}],[3])$. As for the flow measurements, both velocity and shear-stress sensors were used. While velocity measurements are often considered in the literature, due to their widespread use in practice, shear-stress sensors are less common. Nevertheless, they were used here mainly because they are challenging from a numerical point of view, as they involve spatial derivatives of the POD modes. Also, they can be implemented in practice although limitations of accuracy and time resolution may exist (see, for instance, [16]). Different sensor locations were tested, to account for the sensitivity of the proposed approaches to sensor placement. Since the performance of the standard techniques such as LSE or LSQ is influenced by sensor placement, some sensor configurations were selected following the suggestions given for LSE in [6]. On the other hand, none of the considered sensor configurations is optimized for K-LSE or K-LSQ, in order 
to verify the sensitivity of such methods with respect to sensor placement. In fact, optimal sensor placement may turn out to be a time-consuming operation for complex three-dimensional flows.

\subsection{Two-dimensional case: $R e=150$}

For this rather simple flow, we consider the situation in which a limited number of measurements are available, i.e. only 2 sensors. Three different configurations were analyzed, two involving streamwise velocity sensors and one involving shear-stress sensors.

The velocity sensors were placed in relation to the spatial structure of the streamwise component of the first two POD modes. In particular, in the first configuration one streamwise velocity sensor is placed on the maximum of the first POD mode which is closest to the cylinder $(P 1 \simeq(2.39,0.52))$ and one in the middle between P1 and the minimum of the second POD mode closest to the cylinder $(\simeq(1.96,0.50))$. The second configuration has the first streamwise velocity sensor in $\mathrm{P} 1$ and the second one in the point $(1.98,-0.76)$. A third configuration was considered with two shear-stress sensors located on the confining walls $(y= \pm 4.0)$ at $x=4$, in a region which satisfies the following criteria on a shedding cycle: the rms value of the shear-stresses is maximum and the reconstruction error of the shear-stresses is minimum for a given number of POD modes.

The POD low-order model of the two-dimensional flow is obtained by using 95 snapshots, uniformly distributed throughout two vortex shedding cycles $(T \simeq 13$ is the non-dimensional duration of the time interval), and by retaining $N_{r}=6$ modes. The calibration of the model is performed in the same interval using 81 collocation points. As shown in [9], the calibrated model accurately reproduces the flow inside and outside the calibration interval.

Figure 3 shows the POD modal coefficients predicted by K-LSQ and by LSQ considering the third (shear-stress sensors) configuration, together with their reference values found from DNS. In addition, errors in the prediction of the modal coefficients given by LSQ, LSE, QSE, K-LSQ and K-LSE in the first (velocity sensors) and third (shear-stress sensors) configurations are reported in Table1(a) and (b), respectively. The values obtained for the second considered sensor configuration are not shown since they are very similar to those of the first one.

The time interval over which reconstruction is performed is approximately 13 time-units long (non-dimensional time); it contains two shedding cycles, and it starts just after the end of the time interval on which the POD model was built and calibrated.

Tables 1 (a) and (b) show the relative reconstruction errors on the velocity components and on their fluctuating part. It appears that two (velocity or shear-stress) sensors are not sufficient to obtain reliable predictions of the modal coefficients by LSQ, LSE, even if LSE leads to a better estimation than LSQ. Accuracy problems persist also with the QSE approach, even if in this case the predictions are more accurate than those obtained with LSE. The results obtained by LSE and QSE are more accurate in the configuration with velocity sensors because their positions were selected to be effective for LSE, as already pointed out. Nevertheless, the errors on the estimation of the first two modal coefficients are still large. This leads to severe errors in the estimation of the fluctuating part of the velocity field since the first two POD modes represent about $94.8 \%$ of the fluctuating energy. Even if the mean flow energy is important with respect to the fluctuating energy, errors in the modal coefficients lead to detectable errors in the reconstruction of the velocity components. Note that the 


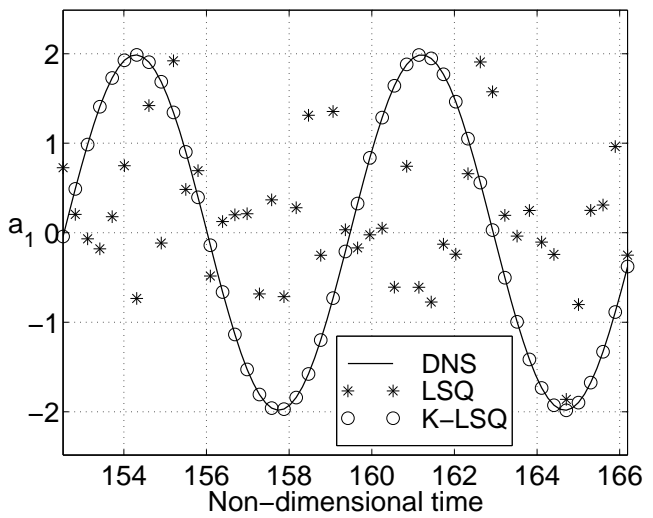

(a)

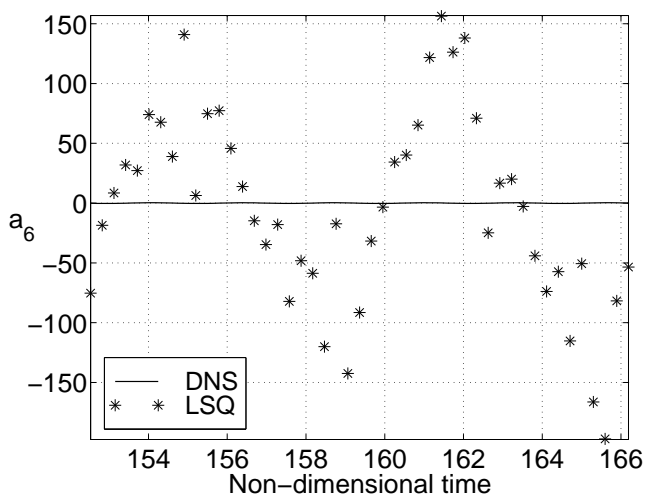

(c)

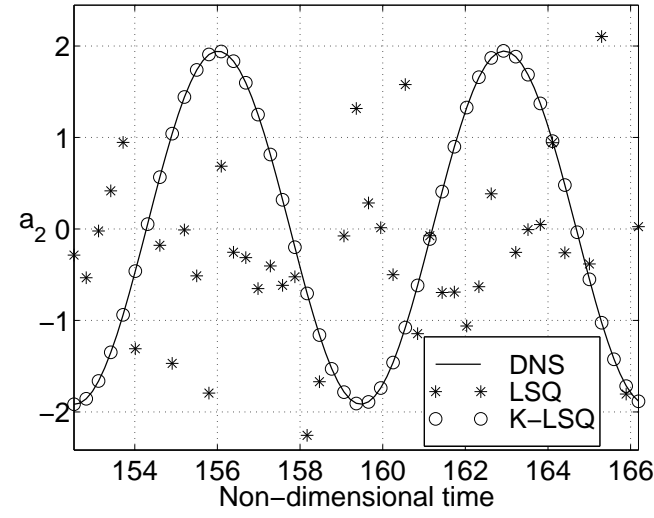

(b)

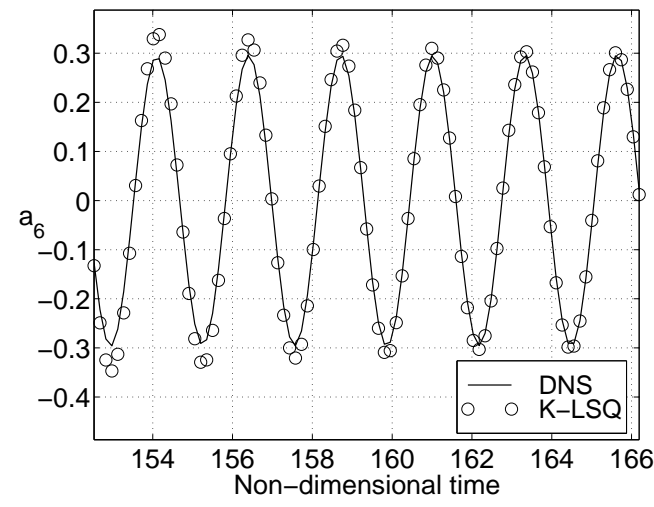

(d)

Figure 3: (a) and (b): POD modal coefficients $a_{1}$ and $a_{2}$ estimated with the K-LSQ and the LSQ approaches, compared to their exact values computed by projection of the DNS velocity fields. (c) coefficient $a_{6}$, DNS and LSQ; (d) coefficient $a_{6}, D N S$ and $K-L S Q$. Note that in (c) and (d) different axis scales are used.

reconstruction errors on the vertical component are larger than those on the streamwise one simply because the contribution of the mean flow on that component is much lower than in the streamwise component. Table 1 shows that both K-LSQ and K-LSE give an accurate estimation not only of the first two modal coefficients, but of all the retained modes. This leads to a precise estimation of the velocity field as well as of its fluctuating part. Moreover, the accuracy of the results is very similar with shear-stress and velocity sensors, indicating a weak sensitivity of the approach with respect to 


\begin{tabular}{|c|c|c|c|c|c|c|}
\hline Tab. 1 $(a)$ & $e\left(a_{1}\right) \%$ & $e\left(a_{2}\right) \%$ & $e\left(a_{3}\right) \%$ & $e\left(a_{4}\right) \%$ & $e\left(a_{5}\right) \%$ & $e\left(a_{6}\right) \%$ \\
\hline LSQ & 108.41 & 340.48 & 538.59 & 2210 & 10900 & 8340 \\
LSE & 71.06 & 27.12 & 99.71 & 97.97 & 99.91 & 99.93 \\
K-LSQ & 0.47 & 0.55 & 2.58 & 2.66 & 4.65 & 4.67 \\
K-LSE & 0.82 & 0.76 & 9.82 & 9.82 & 14.98 & 15.59 \\
\hline \hline Tab.11 b) & $e\left(a_{1}\right) \%$ & $e\left(a_{2}\right) \%$ & $e\left(a_{3}\right) \%$ & $e\left(a_{4}\right) \%$ & $e\left(a_{5}\right) \%$ & $e\left(a_{6}\right) \%$ \\
\hline LSQ & 100.33 & 140.60 & 752.10 & 1040 & 5490 & 37800 \\
LSE & 46.87 & 91.97 & 102.83 & 100.99 & 101.40 & 100.21 \\
K-LSQ & 0.06 & 0.09 & 6.06 & 6.09 & 9.72 & 9.56 \\
K-LSE & 2.99 & 3.08 & 7.07 & 8.31 & 17.99 & 18.47 \\
\hline
\end{tabular}

Table 1: Relative percentage errors (in $L^{2}$ norm) on the estimation of the POD modal coefficients $\left(e\left(a_{i}\right)\right)$ in the first (a) and third (b) sensor configuration. In this case time-averaging is carried out over the estimation time period.

\begin{tabular}{|c|c|c|c|c|}
\hline Tab. 2 $(a)$ & $\overline{e(U)} \%$ & $\overline{e(V) \%}$ & $\overline{e\left(U^{\prime}\right)} \%$ & $\overline{e\left(V^{\prime}\right)} \%$ \\
\hline LSQ & 106.79 & 937.42 & 906.96 & 1370 \\
LSE & 6.32 & 37.14 & 53.96 & 54.26 \\
K-LSQ & 0.63 & 3.97 & 5.39 & 5.80 \\
K-LSE & 0.69 & 4.42 & 5.93 & 6.46 \\
\hline \hline Tab. 2 $(b)$ & $\overline{e(U)} \%$ & $\overline{e(V)} \%$ & $\overline{e\left(U^{\prime}\right)} \%$ & $\overline{e\left(V^{\prime}\right)} \%$ \\
\hline LSQ & 273.22 & 2490 & 2350 & 3640 \\
LSE & 8.05 & 46.14 & 68.32 & 67.44 \\
K-LSQ & 0.65 & 4.10 & 5.54 & 6.00 \\
K-LSE & 0.77 & 4.91 & 6.54 & 7.17 \\
\hline
\end{tabular}

Table 2: Relative percentage errors (in $L^{2}$ norm) on the estimation of the velocity components $\overline{(e(U)}, \overline{e(V)})$ and of their fluctuating part $\left(\overline{e\left(U^{\prime}\right)}, \overline{e\left(V^{\prime}\right)}\right)$, in the first (a) and third (b) sensor configuration. In this case time-averaging is carried out over the estimation time period.

the type and location of the sensors. This is not the case for the LSQ, LSE and QSE methods, which show a higher sensitivity to this aspect, confirming what has already been reported in the literature.

We compare our results to those of [6], Tab. 2(a) 13-th case. With LSE and 2 sensors they found $e\left(a_{1}\right) \simeq 76.6 \%$ and $e\left(a_{2}\right) \simeq 15.1 \%$, errors that are similar to those reported in Table 1 a) for LSE. Using the dynamic estimation, the errors on the same coefficients are two orders of magnitude lower. Furthermore, using K-LSQ method and two shear-stress sensors (Table 1 b) ) the first two modal coefficients are estimated with an error lower than $0.1 \%$, i.e., three orders of magnitude lower than LSE. 


\subsection{Three-dimensional case: $R e=300$}

The flow patterns in this case are definitely more complex than those of the previous one. For this reason, 24 flow measurements were used for the reconstruction procedure, organized in five different configurations, two involving only velocity measurements and three involving both velocity and shear-stress measurements. In the last three configurations, the shear-stress sensors were selected following the same criterion adopted in the $2 \mathrm{D}$ case, they are 14 in number and in all considered cases are symmetrically placed on both the confining walls $(y= \pm 4)$ at $x=4$ and $z=\{1.2,1.5,2.7,3,3.3,4.5,4.8\}$. The placement of the velocity sensors has again been chosen on the basis of the spatial structure of the streamwise velocity of the first 12 POD modes. The different configurations are listed below, together with a brief description of the rationale for the placement of the velocity sensors:

1. 24 velocity sensors distributed on 6 equispaced slices in the axial $(z)$ direction; on each slice, the sensors are on the lines connecting the maximum and minimum closest to the cylinder of the first two POD modes. On each segment, the sensors are approximately in the middle, but slightly closer to the extrema of the first POD mode.

2. 24 velocity sensors distributed on 4 equispaced slices in the axial (z) direction; on each slice, 3 points are selected in the region of overlapping between the maxima and minima of the low-frequency POD modes (modes 3, 4, 7, 8, 9 and 10) and 3 on the overlapping region of the extrema of the vortex shedding modes (modes 1, 2, 5, 6, 7, 11 and 12) (see [3] for details on the separation between low-frequency and vortex-shedding POD modes).

3. 14 shear-stress sensors and 10 velocity sensors distributed on 5 equispaced slices in the axial (z) direction; on each slice, the velocity sensors are placed on the maximum and minimum closest to the cylinder of the first POD mode.

4. 14 shear-stress sensors and 10 velocity sensors. 6 equispaced slices in the axial $(z)$ direction are considered. On 4 slices, 2 velocity sensors are placed as in the previous case. Two sensors are placed on the remaining slices, corresponding respectively to the maximum and minimum of the third POD mode (low frequency mode).

5. 14 shear-stress sensors and 10 velocity sensors located in the wake, at the points reported in table 3 .

The low-order model of the developed three-dimensional flow was derived retaining the first 20 POD modes obtained from a database of 151 snapshots, uniformly distributed over eight vortex shedding cycles ( $\simeq 52$ non-dimensional time units). Calibration was carried out in the same time interval, and the results obtained integrating the dynamic model within the calibration interval are reported in figure2 2 More details of this model can be found in [3] (the model is denoted there as POD2). In contrast to the two-dimensional case, the POD model is inaccurate outside the calibration interval, so it cannot be used as a predictive tool by itself. The estimation of the flow is carried out here in two different time intervals, one starting just after the end of the time interval in which the POD model was calibrated and the other one being about 52 time units distant from the calibration interval. Both the intervals are approximately 30 time units long, including approximately 4 shedding cycles.

RR $n^{\circ} 0123456789$ 


\begin{tabular}{cccc|cccc} 
Velocity sensor & $\mathrm{x}$ & $\mathrm{y}$ & $\mathrm{z}$ & Velocity sensor & $\mathrm{x}$ & $\mathrm{y}$ & $\mathrm{z}$ \\
1 & 5.01 & 1.03 & 2.00 & 6 & 5.01 & -1.03 & 4.00 \\
2 & 6.99 & 1.04 & 2.00 & 7 & 6.99 & -1.04 & 4.00 \\
3 & 6.03 & -1.04 & 2.00 & 8 & 6.03 & 1.04 & 4.00 \\
4 & 6.01 & -0.99 & 2.00 & 9 & 6.01 & 0.99 & 4.00 \\
5 & 5.96 & -0.97 & 2.00 & 10 & 5.96 & 0.97 & 3.99
\end{tabular}

Table 3: Positions of the velocity sensors in the three-dimensional case

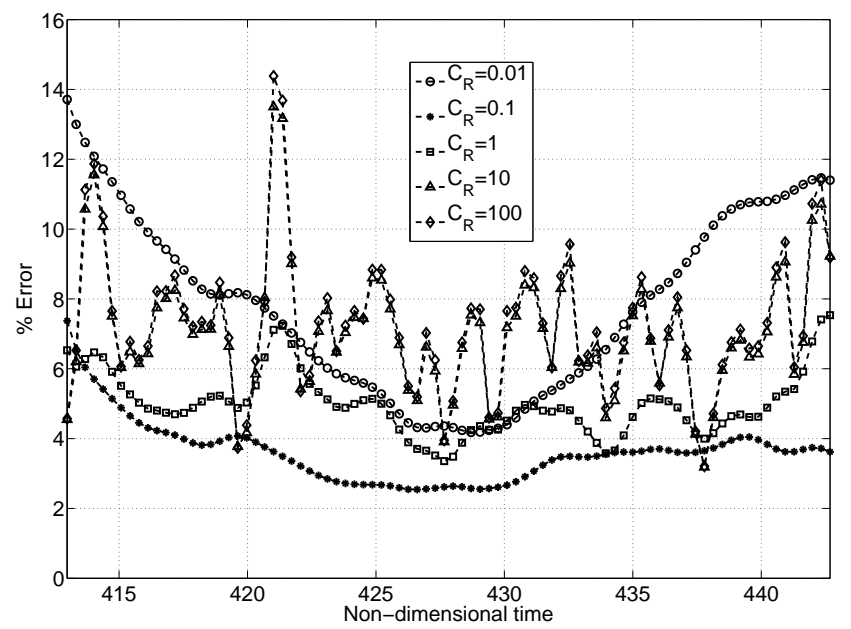

Figure 4: Relative percent error in the reconstruction of the $\mathrm{U}$ component projected on the retained POD modes as a function of time, when varying $C_{R}$. 

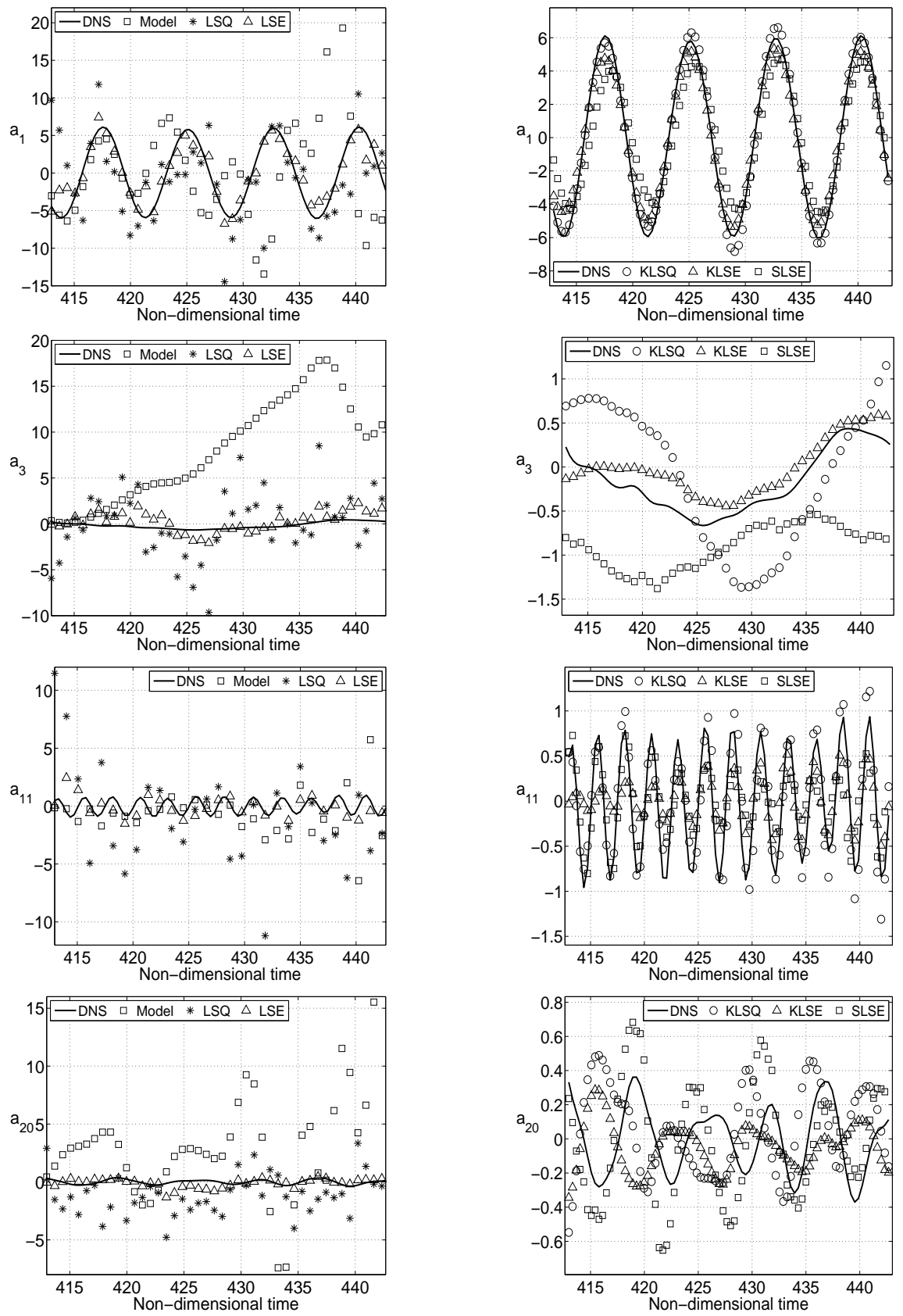

Figure 5: Estimation of some representative modal coefficients in the three-dimensional case, for the configuration (b) (see text) and in the time interval close to the calibration one, together with the reference ones evaluated from the DNS simulation. Note that different axis scales are used on the left and on the right plots.

RR n ${ }^{\circ} 0123456789$ 


\begin{tabular}{|c|c|c|c|}
\hline SC (a) & $\overline{e(U)} \%$ & $\overline{e(V)} \%$ & $\overline{e(W)} \%$ \\
\hline KLSQ & 9.85 & 37.00 & 108.12 \\
KLSE & 9.60 & 36.49 & 103.92 \\
\hline & $\overline{e\left(U^{\prime}\right) \%}$ & $\overline{e\left(V^{\prime}\right) \%}$ & $\overline{e\left(W^{\prime}\right)} \%$ \\
\hline KLSQ & 61.89 & 52.48 & 105.76 \\
KLSE & 9.60 & 36.49 & 103.92 \\
\hline & $\overline{e\left(U_{f}\right)} \%$ & $\overline{e\left(V_{f}\right)} \%$ & $\overline{e\left(W_{f}\right)} \%$ \\
\hline KLSQ & 5.08 & 19.40 & 122.54 \\
KLSE & 3.78 & 15.92 & 86.68 \\
\hline
\end{tabular}

\begin{tabular}{|c|c|c|c|}
\hline SC (b) & $\overline{e(U)} \%$ & $\overline{e(V)} \%$ & $\overline{e(W)} \%$ \\
\hline KLSQ & 10.00 & 36.65 & 111.52 \\
KLSE & 10.03 & 38.19 & 105.57 \\
\hline & $\overline{e\left(U^{\prime}\right) \%}$ & $\overline{e\left(V^{\prime}\right) \%}$ & $\overline{e\left(W^{\prime}\right) \%}$ \\
\hline KLSQ & 62.76 & 51.95 & 109.13 \\
KLSE & 62.93 & 54.10 & 103.27 \\
\hline & $\overline{e\left(U_{f}\right)} \%$ & $\overline{e\left(V_{f}\right)} \%$ & $\overline{e\left(W_{f}\right) \%}$ \\
\hline KLSQ & 4.18 & 15.87 & 96.63 \\
KLSE & 3.61 & 16.50 & 72.35 \\
\hline
\end{tabular}

\begin{tabular}{|c|c|c|c|}
\hline SC (c) & $\overline{e(U)} \%$ & $\overline{e(V)} \%$ & $\overline{e(W)} \%$ \\
\hline KLSQ & 10.36 & 38.47 & 112.72 \\
KLSE & 10.42 & 38.59 & 108.99 \\
\hline & $\overline{e\left(U^{\prime}\right) \%}$ & $\overline{e\left(V^{\prime}\right)} \%$ & $\overline{e\left(W^{\prime}\right) \%}$ \\
\hline KLSQ & 65.02 & 54.55 & 110.30 \\
KLSE & 65.37 & 54.70 & 106.68 \\
\hline & $\overline{e\left(U_{f}\right)} \%$ & $\overline{e\left(V_{f}\right) \%}$ & $\overline{e\left(W_{f}\right)} \%$ \\
\hline KLSQ & 5.61 & 22.97 & 137.30 \\
KLSE & 4.22 & 18.51 & 97.95 \\
\hline
\end{tabular}

\begin{tabular}{|c|c|c|c|}
\hline SC (d) & $\overline{e(U)} \%$ & $\overline{e(V)} \%$ & $\overline{e(W)} \%$ \\
\hline KLSQ & 10.21 & 37.75 & 109.98 \\
KLSE & 10.37 & 38.19 & 108.10 \\
\hline & $\overline{e\left(U^{\prime}\right)} \%$ & $\overline{e\left(V^{\prime}\right)} \%$ & $\overline{e\left(W^{\prime}\right)} \%$ \\
\hline KLSQ & 64.15 & 53.54 & 107.56 \\
KLSE & 65.08 & 54.12 & 105.82 \\
\hline & $e\left(U_{f}\right) \%$ & $\overline{e\left(V_{f}\right)} \%$ & $e\left(W_{f}\right) \%$ \\
\hline KLSQ & 5.02 & 20.16 & 121.39 \\
KLSE & 4.03 & 16.41 & 91.39 \\
\hline
\end{tabular}

\begin{tabular}{|c|c|c|c|}
\hline SC (e) & $\overline{e(U) \%}$ & $\overline{e(V)} \%$ & $\overline{e(W)} \%$ \\
\hline KLSQ & 11.30 & 40.99 & 119.15 \\
KLSE & 10.24 & 38.35 & 107.59 \\
\hline & $\overline{e\left(U^{\prime}\right)} \%$ & $\overline{e\left(V^{\prime}\right)} \%$ & $\overline{e\left(W^{\prime}\right)} \%$ \\
\hline KLSQ & 71.01 & 58.17 & 116.48 \\
KLSE & 64.23 & 54.31 & 105.33 \\
\hline & $\overline{e\left(U_{f}\right)} \%$ & $\overline{e\left(V_{f}\right)} \%$ & $\overline{e\left(W_{f}\right)} \%$ \\
\hline KLSQ & 6.78 & 25.30 & 166.46 \\
KLSE & 3.70 & 16.61 & 87.30 \\
\hline
\end{tabular}

Table 4: Relative percent errors, in $L^{2}$ norm, in the reconstruction of the velocity components $(\overline{e(U)}$, $\overline{e(V)}, \overline{e(W)})$, of their fluctuating part $\left(\overline{e\left(U^{\prime}\right)}, \overline{e\left(V^{\prime}\right)}, \overline{e\left(W^{\prime}\right)}\right)$, and of the part of the velocity field projected on the retained POD modes $\left(\overline{e\left(U_{f}\right)}, \overline{e\left(V_{f}\right)}, \overline{e\left(W_{f}\right)}\right)$. The errors are averaged in time in the estimation interval, which starts just after the calibration interval. SC: Sensor Configuration.

The parameter $C_{R}$ of equation 8 was selected by experimenting different values. For example, in figure 4 we show the $L^{2}$ relative error in the reconstruction of the $\mathrm{U}$ component projected on the retained POD modes, as a function of $C_{R}$. The results are relative to the configuration (b) in the time interval close to the calibration one, using K-LSE. For all of the results shown in the following we took $C_{R}=0.1$ for K-LSE. Note that for $C_{R} \geq 10^{2}$ the results are basically those of a simple LSE. A similar analysis was performed using K-LSQ and the optimal value that was selected is $C_{R}=10$. 
Results relative to the configuration (b) in the time interval close to the calibration one are reported in figure 5, where some representative modal coefficients predicted by the calibrated POD model, LSQ, LSE, K-LSQ, K-LSE and SLSE are plotted, together with the projection of the DNS velocity fields on the corresponding POD mode.

Results of configuration (b) has been shown because the placement of the sensors is appropriate for the LSE method, as already discussed, and this makes the comparison with the proposed approaches more comprehensive. The results obtained with the other sensor configurations and on the time interval far from the calibration one are qualitatively analogous and quantitatively similar to those reported in figure 5, except for the LSE and the LSQ methods which are more sensitive to sensor placement. In figure 5 it is seen that LSE, LSQ, and also the calibrated POD model provide reasonable predictions only for the first two modal coefficients, that are associated with the vortex-shedding dynamics. The predictions of the remaining modes are completely unreliable. When dynamic estimation is applied, or when the SLSE approach is used, predictions are definitely improved. In particular, this is true for modes like $a_{1}$ or $a_{11}$ that are related to the vortex-shedding, i.e., almost periodic with a period that is the same or a multiple of the vortex-shedding period. The prediction of the remaining modes is definitely less accurate (see $a_{3}$ and $a_{20}$ ), especially when very low frequencies are dominant, as in the case of $a_{3}$. However, the overall accuracy is significantly improved in comparison with the LSQ and LSE approaches alone. The K-LSE approach is systematically more accurate than SLSE. This is quantitatively confirmed by the relative error between the DNS velocity components and those reconstructed by the dynamic approaches and the SLSE one, reported in table 4 for all the sensor configurations. However, the table shows that there are no significant differences in accuracy between the K-LSQ, K-LSE and SLSE methods, and the reconstruction errors can be considered satisfactory if we consider the complexity of the flow with respect to the 2D case. Indeed, the streamwise and vertical velocity components are reconstructed with errors of the order of $9 \%$ and $33 \%$, respectively. Note that errors are computed over the whole computational domain, which extends for $20 \mathrm{~L}$ behind the cylinder, and, as it is qualitatively shown in figure 6 the reconstruction errors are small in the near-wake and they progressively increase moving away from the cylinder in the downstream direction. Concerning the spanwise component of the velocity, errors are large since the retained POD modes themselves poorly represent this component of velocity as it is not energetically significant, in average, with respect to the remaining ones. This aspect might be improved working on the construction of the POD basis choosing, for instance, a different norm which weights more the spanwise component of the velocity or which corresponds to a quantity different from kinetic energy. The K-LSQ, K-LSE and SLSE methods are also similar in the sensitivity of the predictions to sensors type and placement, which is generally low. Nevertheless, the predictions given by the K-LSE method are systematically the most insensitive to sensors placement. Table 4 also shows the relative reconstruction errors on the fluctuating part of the velocity components. The errors in the reconstruction of the fluctuating part of both the streamwise and the lateral velocity components are comparable. To help the interpretation of the errors in the reconstruction of the fluctuating flow field, which might seem large at first sight, it is important to point out that, outside the calibration interval, the amount of fluctuating energy that can be recovered by 20 modes is slightly more than $60 \%$ ([3]). Therefore, the error in the velocity components is due in part to a pure approximation error. In other words, the accuracy of the best possible reconstruction 


\begin{tabular}{|c|c|c|c|}
\hline SC (a) & $\overline{e(U)} \%$ & $\overline{e(V)} \%$ & $\overline{e(W)} \%$ \\
\hline KLSQ & 8.79 & 32.56 & 114.51 \\
KLSE & 8.96 & 33.54 & 110.95 \\
\hline & $\overline{e\left(U^{\prime}\right) \%}$ & $\overline{e\left(V^{\prime}\right) \%}$ & $\overline{e\left(W^{\prime}\right)} \%$ \\
\hline KLSQ & 55.91 & 46.13 & 111.51 \\
KLSE & 56.97 & 47.53 & 108.00 \\
\hline & $\overline{e\left(U_{f}\right)} \%$ & $\overline{e\left(V_{f}\right)} \%$ & $\overline{e\left(W_{f}\right)} \%$ \\
\hline KLSQ & 4.13 & 15.74 & 101.87 \\
KLSE & 3.63 & 14.90 & 84.19 \\
\hline
\end{tabular}

\begin{tabular}{|c|c|c|c|}
\hline SC (b) & $\overline{e(U)} \%$ & $\overline{e(V)} \%$ & $\overline{e(W)} \%$ \\
\hline KLSQ & 9.50 & 34.47 & 121.52 \\
KLSE & 8.83 & 33.11 & 111.35 \\
\hline & $\overline{e\left(U^{\prime}\right) \%}$ & $\overline{e\left(V^{\prime}\right) \%}$ & $\overline{e\left(W^{\prime}\right) \%}$ \\
\hline KLSQ & 60.50 & 48.87 & 118.35 \\
KLSE & 56.17 & 46.91 & 108.42 \\
\hline & $\overline{e\left(U_{f}\right)} \%$ & $\overline{e\left(V_{f}\right)} \%$ & $\overline{e\left(W_{f}\right) \%}$ \\
\hline KLSQ & 5.15 & 19.82 & 118.19 \\
KLSE & 3.34 & 14.68 & 80.84 \\
\hline
\end{tabular}

\begin{tabular}{|c|c|c|c|c|c|c|c|}
\hline $\mathrm{SC}(\mathrm{c})$ & $\overline{e(U)} \%$ & $\overline{e(V)} \%$ & $\overline{e(W)} \%$ & $\mathrm{SC}(\mathrm{d})$ & $\overline{e(U)} \%$ & $\overline{e(V)} \%$ & $\overline{e(W)} \%$ \\
\hline KLSQ & $\begin{array}{l}9.47 \\
\end{array}$ & $\begin{array}{l}33.88 \\
\end{array}$ & 120.45 & KLSQ & 9.42 & 35.45 & 121.86 \\
\hline KLSE & 9.17 & 33.19 & 110.95 & KLSE & 9.44 & 34.40 & 110.40 \\
\hline & $\overline{e\left(U^{\prime}\right) \%}$ & $\overline{e\left(V^{\prime}\right) \%}$ & $\overline{e\left(W^{\prime}\right) \%}$ & & $\overline{e\left(U^{\prime}\right) \%}$ & $\overline{e\left(V^{\prime}\right) \%}$ & $\overline{e\left(W^{\prime}\right) \%}$ \\
\hline KLSQ & 60.08 & 47.97 & 117.20 & KLSQ & 59.93 & 50.26 & 118.64 \\
\hline KLSE & 58.36 & 47.03 & 108.09 & KLSE & 59.93 & 48.71 & 107.54 \\
\hline & $\overline{e\left(U_{f}\right) \%}$ & $\overline{e\left(V_{f}\right) \%}$ & $\overline{e\left(W_{f}\right)} \%$ & & $\overline{e\left(U_{f}\right)} \%$ & $\overline{e\left(V_{f}\right) \%}$ & $\overline{e\left(W_{f}\right)} \%$ \\
\hline KLSQ & 5.28 & 19.02 & 133.11 & KLSQ & 5.11 & 20.46 & 133.56 \\
\hline KLSE & 3.07 & 13.56 & 74.63 & KLSE & 3.62 & 15.74 & 76.05 \\
\hline
\end{tabular}

\begin{tabular}{|c|c|c|c|}
\hline SC (e) & $\overline{e(U) \%}$ & $\overline{e(V) \%}$ & $\overline{e(W)} \%$ \\
\hline KLSQ & 10.60 & 38.50 & 136.53 \\
KLSE & 9.22 & 33.49 & 110.24 \\
\hline & $\overline{e\left(U^{\prime}\right)} \%$ & $\overline{e\left(V^{\prime}\right)} \%$ & $\overline{e\left(W^{\prime}\right)} \%$ \\
\hline KLSQ & 67.42 & 54.59 & 132.86 \\
KLSE & 58.65 & 47.45 & 107.38 \\
\hline & $\overline{e\left(U_{f}\right)} \%$ & $\overline{e\left(V_{f}\right)} \%$ & $\overline{e\left(W_{f}\right)} \%$ \\
\hline KLSQ & 7.29 & 26.00 & 183.25 \\
KLSE & 3.27 & 14.10 & 77.47 \\
\hline
\end{tabular}

Table 5: Relative percent errors, in $L^{2}$ norm, in the reconstruction of the velocity components $(\overline{e(U)}$, $\overline{e(V)}, \overline{e(W)}$ ), of their fluctuating part $\left(\overline{e\left(U^{\prime}\right)}, \overline{e\left(V^{\prime}\right)}, \overline{e\left(W^{\prime}\right)}\right)$, and of the part of the velocity field projected on the retained POD modes $\left(\overline{e\left(U_{f}\right)}, \overline{e\left(V_{f}\right)}, \overline{e\left(W_{f}\right)}\right)$. The errors are averaged in time in the estimation interval, which is about 52 time units far from the calibration interval.

is limited from above by the capability of the POD modes to actually represent the flow outside the time interval where the snapshots were taken, which however, increases using a larger snapshots database, as shown in [3].

In order to separate the accuracy of the estimation methods from the representativeness of the POD basis, we evaluated the errors between the estimated field and the projections of the velocity fields on the retained POD modes. Such errors are reported in tables 4 and 5 . These errors are 

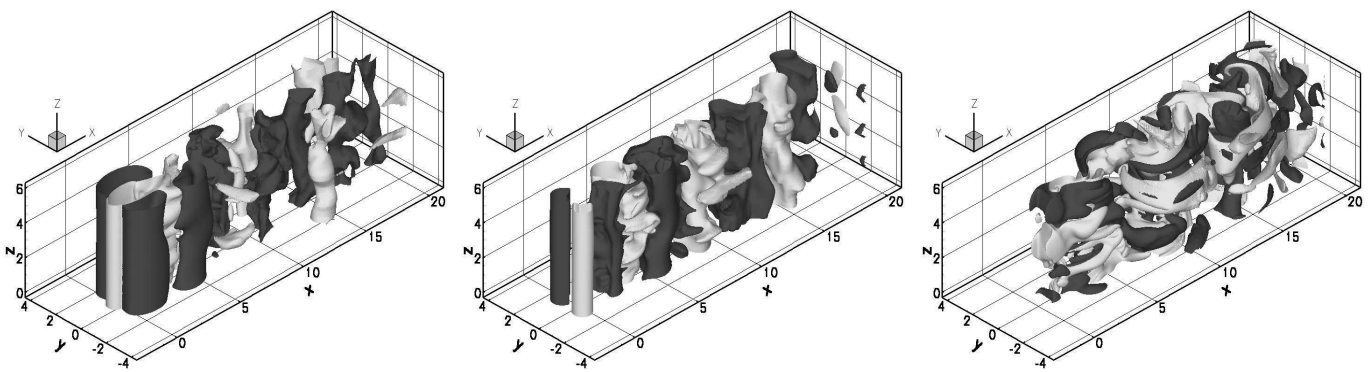

(a)
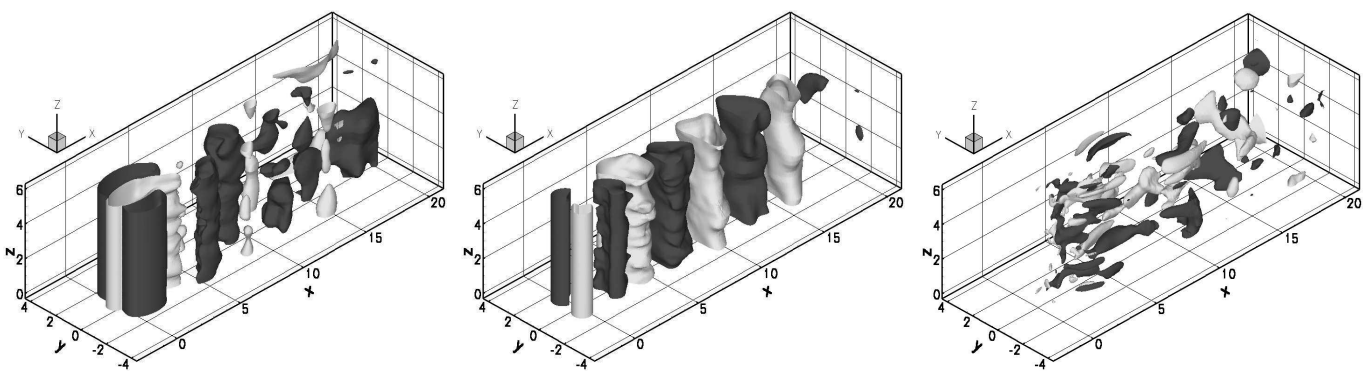

(b)
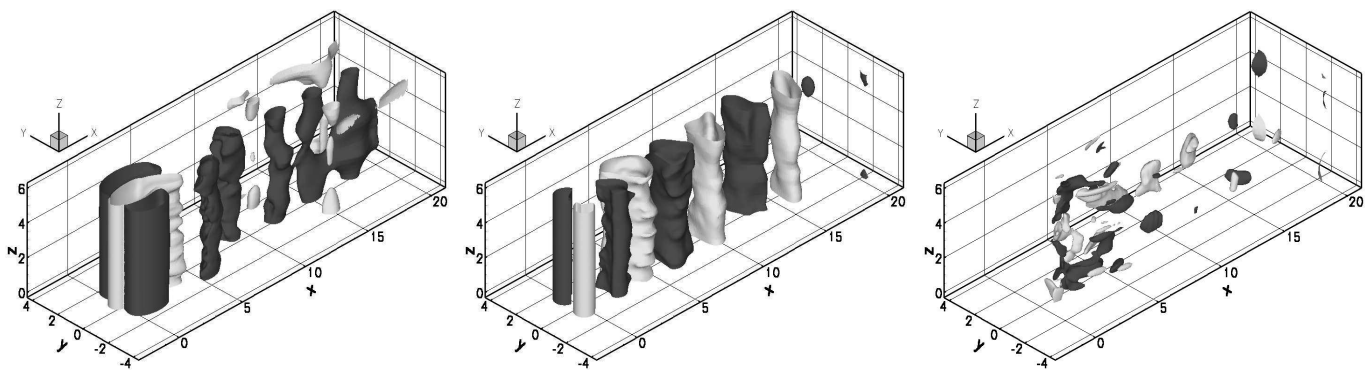

(c)

Figure 6: Isosurfaces of the velocity components $u$ (left, grey $=0.5$, dark grey $=1.0$ ), $v$ (center, grey $=-0.25$, dark grey $=0.25$ ) and $w$ (right, grey $=-0.075$, dark grey $=0.075$ ) of a snapshot outside the database: (a) actual snapshot, (b) snapshot projected on the retained POD modes,(c) reconstructed snapshot using the K-LSE technique with the sensor configuration (b).

systematically and significantly lower than those observed in the reconstruction of the whole flow fields.

Comparing the results reported in table 4 with those of table 5 where the same quantities are shown for the time interval far from the calibration one, it can be seen that the accuracy of the 
predictions is independent of the distance from the calibration interval, this being a very positive feature of all the proposed approaches.

In figure 6 the velocity components obtained by DNS at $t=426.6$ (a snapshot outside the database used for the derivation and calibration of the POD model) are plotted together with their projection in the space of the retained POD basis, which represents the best approximation of the flow which can be estimated with the retained POD modes, and with the prediction given by the K-LSE method. It can be seen that the main structures characterizing the streamwise and lateral velocity fields are well reconstructed. As for the spanwise velocity component, the reconstruction accuracy is not satisfactory, but this is due to the fact that it is one order of magnitude lower than the other components, as already discussed.

\section{Conclusions}

We devised a method to construct a non-linear observer for unsteady flows. This method is based on the coupling of a non-linear low-dimensional model of the flow with a linear technique that estimates the coefficients of the flow representation in terms of POD modes. The underlying idea is that the estimated flow should approximately satisfy the POD model. The coupling leads to a nonlinear minimization problem solved by a pseudo-spectral approach and a Newton method.

The non-linear observer was applied to the laminar flow around a confined square cylinder at two different Reynolds numbers; at the first the flow is two-dimensional, while in the second case threedimensional phenomena occur in the wake. In the two-dimensional case, since the flow patterns are rather simple, the results show that the proposed procedure is able to give a significantly more accurate estimate of the POD coefficients even with a limited number of sensors, than those obtained with the LSQ and the LSE approaches. The QSE method improves the predictions of the LSE, even if the overall accuracy remains definitely lower than those given by the proposed approaches. In the three-dimensional case, the flow dynamics is more complex, and not only LSE and LSQ, but also the calibrated POD dynamical system provide unsatisfactory coefficient estimations when used outside the calibration interval. Conversely, the proposed procedure, combined with either LSQ or LSE, gives accurate predictions of the coefficients of those POD modes that are related to vortex shedding. For the remaining modes, the accuracy is lower. Nevertheless, the instantaneous velocity fields are reconstructed with satisfactory accuracy, both close and far from the calibration interval of the POD model. Moreover, K-LSE and K-LSQ methods are weakly sensitive to sensor type and placement. The results obtained with the proposed approaches are comparable to those obtained by the SLSE approach, which also uses the temporal history of the flow measurements, but in the Fourier space. However, this latter techniques has computational complexity which is significantly larger than that of LSE or LSQ, and it is comparable to that of the proposed approaches.

This work was funded in part by the HPC-EUROPA project (RII3-CT-2003-506079). IDRIS (Orsay, France) and M3PEC (Université Bordeaux 1, France) provided the computational resources. 


\section{References}

[1] R. J. Adrian. On the role of conditional averages in turbulent theory. In G. Patteson and J. Zakin, editors, Turbulence in Liquids: Proceedings of the 4th Biennial Symposium on Turbulence in Liquids, pages 322-332, Princeton, 1977. Science Press.

[2] J.P. Bonnet, D. Cole, J. Delville, M. Glauser, and L. Ukeiley. Stochastic estimation and Proper Orthogonal Decomposition: complementary techniques for identifying structures. Experiments in fluids, 17:307-314, 1994.

[3] M. Buffoni, S. Camarri, A. Iollo, and M.V. Salvetti. Low-dimensional modelling of a confined three-dimensional wake flow. Journal of Fluid Mechanics, 569:141-150, 2006.

[4] S. Camarri and F. Giannetti. On the inversion of the Kármán street in the wake of a confined square cylinder. Accepted for publication on J. Fluid Mech, 2006.

[5] K. Cohen, S. Siegel, and T. McLaughlin. A heuristic approach to effective sensor placement for modeling of a cylinder wake. Computers and Fluids, 35:103-120, 2006.

[6] K. Cohen, S. Siegel, D. Wetlesen, J. Cameron, and A. Sick. Effective sensor placements for the estimation of proper orthogonal decomposition mode coefficients in von Kármán vortex street. Journal of Vibration Control, 10:1857-1880, 2004.

[7] D. Ewing and J. Citriniti. Examination of a lse/pod complementary technique using single and multi-time information in the axisymmetric shear layer. In Sorensen, Hopfinger, and Aubry, editors, Proceedings of the IUTAM Symposium on simulation and identification of organized structures in flows, volume 52 of Fluid Mechanics and Its Applications, pages 375-384. Springer, 25-29 May 19971999.

[8] B. Galletti, A. Bottaro, CH. Bruneau, and A. Iollo. Accurate model reduction of transient flows. European Journal of Mechanics / B Fluids, 2006. To appear. Available on-line.

[9] B. Galletti, C. H. Bruneau, L. Zannetti, and A. Iollo. Low-order modelling of laminar flow regimes past a confined square cylinder. Journal of Fluid Mechanics, 503:161-170, 2004.

[10] J. Hoepffner, M. Chevalier, T.R. Bewley, and D.S. Henningson. State estimation in wallbounded flow systems. Part 1. Perturbed laminar flows. Journal of Fluid Mechanics, 534:263294, 2005.

[11] R. E. Kalman. A new approach to linear filtering and prediction problems. Transactions of the ASME-Journal of Basic Engineering, 82(Series D):35-45, 1960.

[12] J. L. Lumley. The structure of inhomogeneous turbulent flows. In Atmospheric Turbulence and Radio Wave Propagation, edited by A. M. Yaglom and V. L. Tatarski, Nauka, Moscow, pages 166-178, 1967.

$\mathrm{RR} \mathrm{n}^{\circ} 0123456789$ 
[13] A. M. Naguib, C. E. Wark, and O. Juckenhöfel. Stochastic estimation and flow sources associated with surface pressure events in a turbulent boundary layer. Physics of Fluids, 13:26112626, September 2001.

[14] R.F. Schmit and M. N. Glauser. Use of low-dimensional methods for wake flowfield estimation from dynamic strain. AIAA Journal, 43(5):1133-1136, 2005.

[15] L. Sirovich. Turbulence and the dynamics of coherent structures. Parts I,II and III. Quarterly of Applied Mathematics, XLV:561-590, 1987.

[16] P.G. Spazzini, G. Iuso, M. Onorato, and N. Zurlo. Design, test and validation of a probe for time-resolved measurement of skin friction. Measurement Science and Technology, 10(7):631639, 1999.

[17] C.E. Tinney, F. Coiffet, J. Delville, A.M. Hall, P. Jordan, and M.N. Glauser. On spectral linear stochastic estimation. Experiments in Fluids, 45(5):763-775, November 2006.

[18] D. Venturi and G.E. Karniadakis. Gappy data and reconstruction procedures for flow past a cylinder. Journal of Fluid Mechanics, 519:315-336, 2004.

[19] K. Willcox. Unsteady flow sensing and estimation via the gappy proper orthogonal decomposition. Computers and Fluids, 35:208-226, 2006. 


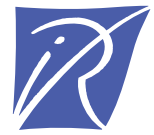

Unité de recherche INRIA Futurs

Parc Club Orsay Université - ZAC des Vignes

4, rue Jacques Monod - 91893 ORSAY Cedex (France)

Unité de recherche INRIA Lorraine : LORIA, Technopôle de Nancy-Brabois - Campus scientifique 615, rue du Jardin Botanique - BP 101 - 54602 Villers-lès-Nancy Cedex (France)

Unité de recherche INRIA Rennes : IRISA, Campus universitaire de Beaulieu - 35042 Rennes Cedex (France)

Unité de recherche INRIA Rhône-Alpes : 655, avenue de l'Europe - 38334 Montbonnot Saint-Ismier (France) Unité de recherche INRIA Rocquencourt : Domaine de Voluceau - Rocquencourt - BP 105 - 78153 Le Chesnay Cedex (France)

Unité de recherche INRIA Sophia Antipolis : 2004, route des Lucioles - BP 93 - 06902 Sophia Antipolis Cedex (France) 


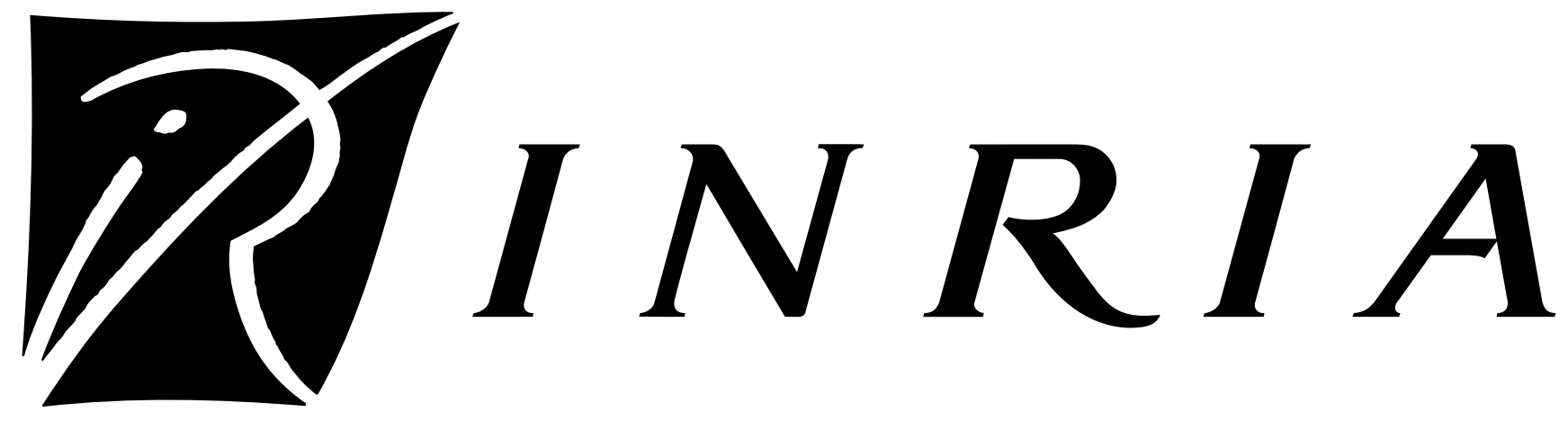

